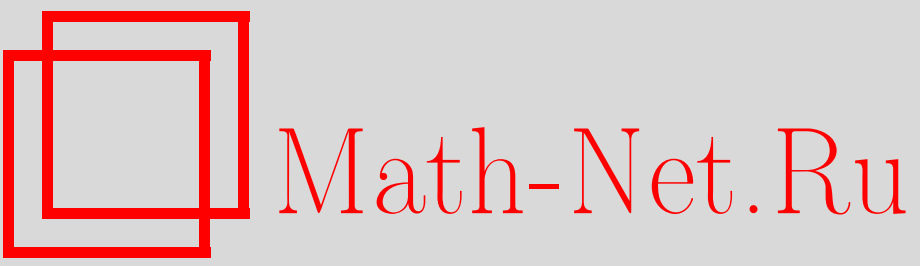

А. О. Иванов, А. А. Тужилин, Структура множества плоских минимальных сетей с заданными топологией и границей, УМH, 1996, том 51, выпуск 3, 201202

DOI: https://doi.org/10.4213/rm984

Использование Общероссийского математического портала Math-Net.Ru подразумевает, что вы прочитали и согласны с пользовательским соглашением http://www.mathnet.ru/rus/agreement

Параметры загрузки:

IP: 18.234 .197 .8

26 апреля 2023 г., $14: 36: 21$ 


\title{
СТРУКТУРА МНОЖЕСТВА ПЛОСКИХ МИНИМАЛЬНЫХ СЕТЕЙ С ЗАДАННЫМИ ТОПОЛОГИЕЙ И ГРАНИЦЕЙ
}

\author{
А.О. Иванов, А. А. ТУЖилин
}

Назовем обобщенной сетью произвольное непрерывное отображение Г связного конечного одномерного клеточного комплекса $G$ (топологического графа) в плоскость $\mathbb{R}^{2}$. При этом граф $G$ будем называть топологией обобщенной сети $\Gamma$. Ограничения отображения $\Gamma$ на нульмерные и одномерные клетки графа $G$ называются, соответственно, вершинами и ребрами обобщенной сети $Г$. Обобщенная сеть, все ребра которой-вложенные регулярные кривые, назьвается просто сетью. Границей $\varphi$ обобщенной сети $Г$ назовем ограничение отображения $\Gamma$ на некоторое фиксированное подмножество $V$ множества вершин графа $G$. Если $M=\varphi(V) \subset \mathbb{R}^{2}$ - образ границы $\varphi$, то будем говорить, что обобщенная сеть $\Gamma$ затягивает множество $M$ по граничному отображению $\varphi$.

Определим длину сети как сумму длин всех ее ребер. Тогда естественно возникает обобщенная проблема Штейнера, состоящая в поиске среди всех сетей, затягивающих некоторое фиксированное конечное множество $M$ точек плоскости, сетей минимальной длины. В данной работе под минимальностью мы понимаем локальную минимальность, которая, напомним, означает, что каждый достаточно малый фрагмент сети является абсолютно минимальной сетью. Другими словами, никакая деформация такой сети, сохраняющая ее границу и малая по носителю и по амплитуде, не уменьшает длину сети. Локальное устройство таких сетей хорошо известно (см. [1], [2]). А именно, все ребра локально минимальной сети представляют собой отрезки, стыкующиеся под углами, больше или равными $120^{\circ}$, в частности, степени вершин такой сети не превосходят трех. При этом можно предполагать, что граница минимальной сети состоит в точности из всех ее вершин степеней 1 и 2 , что мы и будем делать. Сеть $Г$ с гранищей $\varphi$, степени вершин которой не превосходят 3 , и все вершины степеней 1 и 2 являются граничными, назовем сетью IIтейнера. Соответствующие параметризуюшие графы будем называть графами IIтейнера.

Если ограничиться рассмотрением минимальных сетей без циклов, т.е. деревьев, - то хорошо известен алгоритм Мелзака-Хванга (см. [3], [4]), позволяющий для каждой такой топологии $G$ и каждого граничного отображения или построить соответствующую локально минимальную сеть, или убедиться в том, что ее не существует. Одним из центральных наблюдений, позволивших добиться быстрой работы этого алгоритма, является теорема Хванга [4] о единственности минимальной сети данной топологии с данной гранищей. Цель настоящей работы - обобщить результат Хванга на случай минимальных сетей с циклами, которые, как известно, также часто встречаются в приложениях (см. [5], [6]).

Обобщенную сеть назовем линейной сетью, если все ее ребра представляют собой прямолинейные отрезки (возможно, вырожденные). Обозначим через $[G, \varphi]$ конфигурационное пространство $\mathbb{R}^{2 s}$ всех подвижных вершин линейных сетей фиксированной топологии $G$ с заданной границей $\varphi$. Пусть $[G, \varphi]_{\min }$ - множество всех минимумов функционала длины на $[G, \varphi]$. Ясно, что множество $\mathscr{M}_{G}(\varphi)$ всех минимальных сетей топологии $G$ с границей $\varphi$ содержится в $[G, \varphi]_{\min }$.

Нам также понадобятся следующие определения. Ребро сети назовем циклическим, если оно принадлежит какому-нибудь циклу этой сети. Ребро, не входящее ни в один цикл, но инцидентное некоторому циклическому ребру, назовем ребром первого уровня, а все остальные не циклические ребра назовем ребрами второго уровня. Итак, имеет место следующий результат.

Теорема. Если $k(G)$ - чикломатическое число графа ШІтейнера $G$, а множество $\mathscr{M}_{G}(\varphi)$ не пусто, то оно совпадает с внутренностью множества $[G, \varphi]_{\min }$, которое, в свою очередь, является выпуклым телом в $k(G)$-мерном подпространстве из $[G, \varphi]$. В частности, все сети из $\mathscr{M}_{G}(\varphi)$ параллельньи, имеют одинаковую длину и могут быть продеформированы друг в друга в классе локально минимальных сетей того жсе

Работа выполнена при частичной поддержке Российского фонда фундаментальных исследований, Международной Ассоциации по содействию сотрудничеству с учеными Независимых Государств бывшего Советского Союза (INTAS) и гранта Международного центра теоретической физики (ICTP), Италия. 
типа и с той же границей. Более того, при каждой такой деформации остаются неподвижными все ребра второго уровня и все прямые, содержащие ребра первого уровня.

Авторы надеются, что данная теорема позволит в ближайшее время создать алгоритм, которьй для произвольного графа Штейнера $G$ и произвольного граничного отображения $\varphi$ или строит локально минимальную сеть из $\mathscr{M}_{G}(\varphi)$ (какую-нибудь), или определяет, что множество $\mathscr{M}_{G}(\varphi)$ пусто.

Авторы пользуются случаем выразить свою глубокую и искреннюю признательность академику РАН А.Т. Фоменко за его постоянное внимание к работе.

\section{СПИСОК ЛИТЕРАТУРЫ}

[1] Иванов А.О., Тужилин А. А. // Матем. сб. 1991. Т. 182. №12. С. 1813-1844. [2] Ivanov A.O., Tuzhilin A. A. Minimal Networks. Steiner Problem and Its Generalizations. CRC, 1994. [3] Melzak Z. A. // Canad. Math. Bull. 1960. V. 4. P. 143-148. [4] Hwang F. K. // Oper. Res. Lett. 1986. V. 5. P. 235-237. [5] Тужилин А. А., Фоменко А. Т. Элементы геометрии топологии минимальных поверхностей. М.: Наука, 1991. [6] Фоменко А. Т. Топологические вариационные задачи. М.: Изд-во МГУ, 1984.

Московский государственный университет им. М. В. Ломоносова
Принято редколлегией 11.03.1996 